relapsing-GCA were analyzed, these differences were maintained, except for the mean time from GCA diagnosis and the prevalence of ischemic optic neuropathy. Data on remissions were not available in this subgroup of GiACTA patients.

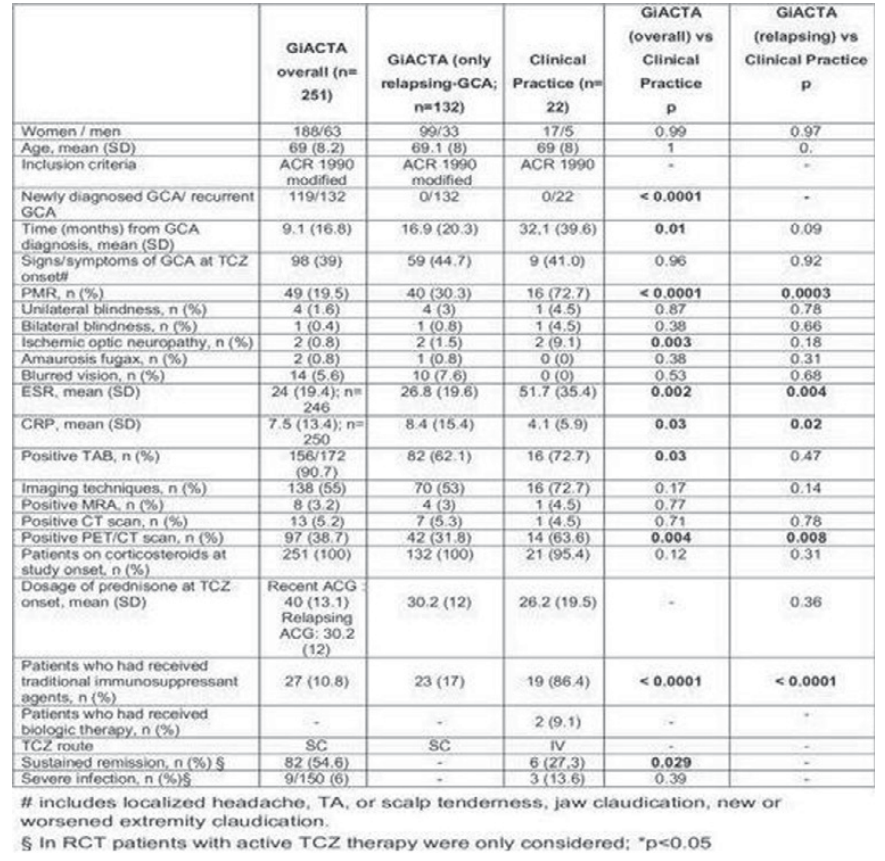

Conclusions: Patients receiving TCZ in the clinical practice study have several baseline clinical and laboratory differences with regard to those included in the GiACTA trial and, therefore, data of this trial should be taken cautiously when applied in a real-world scenario.

References:

[1] Stone J et al. Arthritis Rheumatol.2016; 68 (suppl 10). http://acrabstracts.org/ abstract/efficacy-and-safety-of-tocilizumab-in-patients-with-giant-cell-arteritisprimary-and-secondary-outcomes-from-a-phase-3-randomized-double-blindplacebo-controlled-trial/.

[2] Tuckwell K et al. Semin Arthritis Rheum. 2016 Nov 15. pii: S00490172(16)30275-X

[3] Loricera J et al. Semin Arthritis Rheum. 2015; 44: 717-23.

Disclosure of Interest: None declared

DOI: 10.1136/annrheumdis-2017-eular.3489

\section{OP0136 MICRORNA-223-3P EXPRESSION IN AFFECTED SKIN OF ADULT IGA VASCULITIS CORRELATES WITH THE SEVERITY OF SKIN INVOLVEMENT}

A. Hocevar ${ }^{1}$, J. Pizem ${ }^{2}$, M. Tomsic ${ }^{1}$, D. Glavac ${ }^{2} .{ }^{1}$ Department of Rheumatology, University Medical Centre Ljubljana; ${ }^{2}$ Institute of Pathology, Faculty of Medicine, University of Ljubljana, Ljubljana, Slovenia

Background: IgA vasculitis (IgAV) represents a common systemic vasculitis in paediatric and adult population. Our current knowledge of disease pathogenesis is still very limited and there is no information on miRNAs profile in IgAV.

Objectives: The aim of our study was to determine the expression of three miRNAs (miR-148-3p, miR-155-5p and miR-223-3p) in the affected skin of adult IgAV patients.

Methods: The study included 65 skin samples from consecutive, untreated IgAV patients $(61 \%$ male, median age 67.6 years, range 29-91), diagnosed between October 2014 and September 2016, and 10 control skin samples. Total RNA was isolated from tissue section of formalin-fixed, paraffin-embedded samples of biopsied IgAV vasculitic skin lesions and normal skin samples. Expression of miR-148-3p, miR-155-5p and miR-223-3p was measured using qRT-PCR. Skin miRNAs expression was then correlated to clinical characteristics of adult IgAV patients. To present relative miRNA expression the $\triangle \Delta$ CT method was used. Results: We found significantly higher expression levels of miR-223-3p in the affected skin compared to controls (14-fold; $p<0.001$ ). The expression of the 148b$3 p$ and miR-155-5p was near normal levels (1.05-fold and 1.13-fold increase, respectively). The differences in the expression of miR-223-3p depending on clinical parameters of IgAV are presented in Table 1. Patients with necrotic skin lesions had significantly higher miR-223 tissue expression than those with non-necrotic purpura $(p=0.020)$. Gastrointestinal tract $(G I T)$ involvement inversely correlated with the level of skin miR-223 expression $(p=0.024)$. No significant relationship between renal involvement and skin miR-223 was found.

Conclusions: miR-223 expression was increased in the affected skin of IgAV in comparison to normal skin. Levels of miR-223 expression correlated with severity of skin involvement and inversely with GIT involvement.
Table 1. miR-223-3p expression in IgAV

\begin{tabular}{|c|c|c|c|c|c|c|}
\hline \multirow[t]{2}{*}{ Characteristics } & & \multirow[t]{2}{*}{ Number of cases } & \multicolumn{3}{|c|}{$\Delta \Delta C T$ miR223-3p } & \multirow[t]{2}{*}{$P$ value } \\
\hline & & & median & IQR1 & IQR2 & \\
\hline \multirow[t]{2}{*}{ General symptoms } & YES & 10 & 3.11 & 1.86 & 5.55 & 0.683 \\
\hline & NO & 55 & 3.72 & 2.53 & 5.38 & \\
\hline \multirow[t]{2}{*}{ Arthritis } & YES & 6 & 3.04 & 2.67 & 4.60 & 0.482 \\
\hline & NO & 59 & 3.72 & 2.48 & 4.60 & \\
\hline \multirow[t]{2}{*}{ Generalized purpura } & YES & 37 & 3.72 & 2.46 & 5.87 & 0.615 \\
\hline & NO & 28 & 3.63 & 2.56 & 5.10 & \\
\hline \multirow[t]{2}{*}{ Skin necroses } & YES & 32 & 4.68 & 2.94 & 5.84 & 0.020 \\
\hline & NO & 33 & 3.19 & 1.97 & 4.76 & \\
\hline \multirow[t]{2}{*}{ GIT involvement } & YES & 16 & 2.78 & 1.69 & 3.90 & 0.024 \\
\hline & NO & 49 & 4.29 & 2.69 & 5.62 & \\
\hline \multirow[t]{2}{*}{ Severe GIT involvement } & YES & 5 & 2.67 & 1.88 & 3.07 & 0.078 \\
\hline & NO & 60 & 4.22 & 2.56 & 5.57 & \\
\hline \multirow[t]{2}{*}{ Renal involvement } & YES & 28 & 4.29 & 2.69 & 5.72 & 0.260 \\
\hline & NO & 37 & 3.20 & 1.82 & 5.41 & \\
\hline \multirow[t]{2}{*}{ Severe renal involvement } & YES & 9 & 4.50 & 2.44 & 5.13 & 0.955 \\
\hline & NO & 56 & 3.60 & 2.49 & 5.50 & \\
\hline \multirow[t]{2}{*}{ Elevated serum IgA level } & YES & 30 & 4.63 & 2.71 & 5.93 & 0.041 \\
\hline & NO & 35 & 3.19 & 1.75 & 4.80 & \\
\hline
\end{tabular}

Legend: generalized purpura - purpura above the waist; GIT - gastrointestinal tract; severe GIT involvement - bloody diarrhoea or ileus or surgical intervention; severe renal involvement - acute kidney injury or nephrotic syndrome.

Disclosure of Interest: None declared

DOI: 10.1136/annrheumdis-2017-eular.2250

\section{OP0137 AUTO-REACTIVE B CELLS ESCAPE PERIPHERAL TOLERANCE CHECKPOINTS IN PATIENTS WITH PR3-ANCA ASSOCIATED VASCULITIS}

$\underline{D . ~ C o r n e c ~}^{1,2}$, A. Hummel ${ }^{1}$, T. Peikert ${ }^{1}$, J.-O. Pers ${ }^{2}$, U. Specks ${ }^{1}$. ${ }^{1}$ Mayo Clinic, Rochester MN, United States; ${ }^{2} \mathrm{CHRU}$ and University, Brest, France

Background: While extensive studies have been performed to characterize ANCA, little is known about the auto-reactive B cells that produce these autoantibodies. Indirect evidence previously suggested the presence of circulating PR3-specific B cells in patients with PR3-ANCA-associated vasculitis (AAV).

Objectives: To develop a method to detect circulating PR3-specific B cells in patients with PR3-AAV, to study their proportion among the different B-cell subsets and to assess their relationship with disease activity.

Methods: An enzymatically inactive, conformationally mature, recombinant PR3 (rPR3) was tagged using FITC or biotin. To study the ability of this rPR3 to bind specifically to cells expressing PR3-specific immunoglobulins on their surface, we used two hybridoma cell lines, MCPR3-2 (producing an anti-human PR3 monoclonal antibody) and MCPR3-13 (producing an anti-mouse PR3 monoclonal antibody, with no cross-reactivity with human PR3). We measured the proportion of PR3-FITC positive B cells among PBMCs in 13 patients with PR3-AAV and 14 healthy controls (HCs) by flow cytometry. We then developed a multi-color flow cytometry including CD19, IgD, CD27, CD38, CD24 and biotinylated rPR3 to measure the proportion of PR3-specific B cells among different B-cell subsets in an independent group of 13 patients with PR3-AAV and $11 \mathrm{HCs}$.

Results: rPR3 efficiently bound MCPR3-2 hybridoma cells but not MCPR3-13. Specificity of the staining was confirmed by competition experiments: preincubation of MCPR3-2 cells with untagged human rPR3 totally abrogated rPR3-FITC staining, whereas pre-incubation with mouse rPR3 had no effect. Dose-ranging experiments defined the optimal concentration of rPR3 to stain cells expressing anti-PR3 immunoglobulin. The mean (SEM) proportion of rPR3-FITC stained B cells was higher in patients with PR3-AAV compared to HCs: $2.10 \%$ (2.33) vs $0.45 \%(0.19)$ respectively, $\mathrm{p}<0.001$. Patients with active disease had numerically higher proportions of PR3-specific $B$ cells than patients in remission: $3.66 \%(3.28)$ vs $1.10 \%(0.52), p=0.09$. In HCs, the proportion of PR3-specific $\mathrm{B}$ cells was highest among the transitional B-cell subset, and decreased along with the maturation of $B$ cells (figure). Conversely, in patients, the proportion of PR3-specific B cells progressively increased with the maturation of B cells (median $1.9 \%$ of naïve B cells, $2.30 \%$ of IgD+ memory B cells, $2.37 \%$ of IgDmemory $\mathrm{B}$ cells, and $3.68 \%$ of plasmablasts, $\mathrm{p}<0.05$ for all comparisons with the naïve subset).
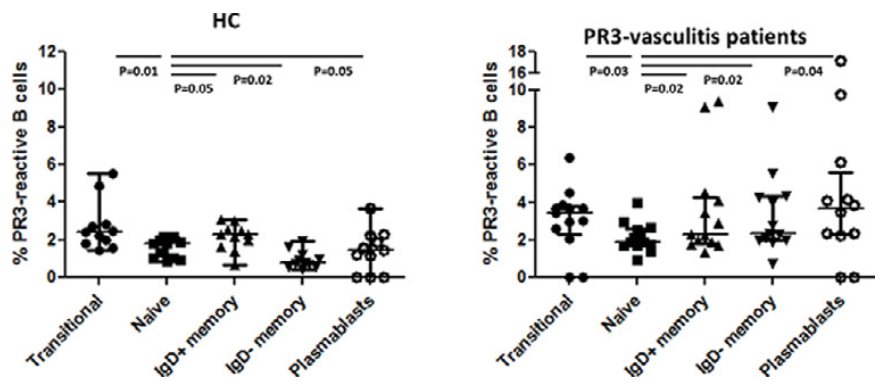

Conclusions: This study describes an original method to detect and study 
circulating auto-reactive $B$ cells in patients with PR3-AAV, and suggests that PR3-specific $B$ cells are associated with disease activity and may represent a promising biomarker to predict relapse risk in patients in clinical remission. The progressive enrichment in PR3-specific B cells during the B-cell maturation steps in patients suggest that auto-reactive B cells are actively selected and escape peripheral tolerance checkpoints.

Disclosure of Interest: None declared

DOI: 10.1136/annrheumdis-2017-eular.6505

\section{THURSDAY, 15 JUNE 2017 \\ HPR mind over matter - patients perspectives}

\section{OP0138-HPR DO PATIENTS' TREATMENT BELIEFS AFFECT TREATMENT CHOICES IN KNEE AND HIP OSTEOARTHRITIS?}

E.M. Selten ${ }^{1}$, R. Geenen ${ }^{2}$, H.J. Schers ${ }^{3}$, F.H. van den Hoogen ${ }^{1}$, R.G. van der Meulen-Dilling ${ }^{4}$, W.H. van der Laan ${ }^{5}$, M.W. Nijhof ${ }^{6}$, C.H. van den Ende ${ }^{1}$,

J.E. Vriezekolk ${ }^{1} .{ }^{1}$ Rheumatology, Sint Maartenskliniek, Nijmegen; ${ }^{2}$ Psychology, Utrecht University, Utrecht; ${ }^{3}$ Primary and Community Care, Radboud University Nijmegen Medical Centre, Nijmegen; ${ }^{4}$ Physical Therapy and Manual Therapy, Partnership Velperweg, Arnhem; ${ }^{5}$ Rheumatology, Sint Maartenskliniek, Woerden; ${ }^{6}$ Orthopaedics, Sint Maartenskliniek, Nijmegen, Netherlands

Background: Patients' beliefs about treatment modalities for knee/hip osteoarthritis $(\mathrm{OA})$ might influence their treatment choices. The Theory of Planned Behavior predicts that patients' beliefs, the norms and values of one's social environment (subjective norm) and one's perceived self-efficacy influence behaviour ${ }^{1}$ Moreover, symptom severity may influence treatment choices ${ }^{2}$. However, these relationships have not been studied yet in the context of treatment decisionmaking

Objectives: To test whether treatment beliefs, subjective norm, perceived selfefficacy and symptom severity were associated with intended treatment choices in $\mathrm{OA}$.

Methods: Patients with knee/hip OA who visited the Sint Maartenskliniek in 2015 and $2016(\mathrm{~N}=700)$ were invited to fill out a booklet. The Treatment beliefs in OsteoArthritis questionnaire was used to assess positive and negative treatment beliefs regarding five treatment modalities: physical activities, pain medication, physiotherapy, injections and arthroplasty. Other measures were demographic and clinical variables, self-efficacy (ASES), and symptom severity (WOMAC). Associations between variables were assessed in three models (Figure 1): 1) whether treatment beliefs are associated with intended treatment choice (model 1); 2) whether treatment beliefs, subjective norm and perceived self-efficacy are associated with intended treatment choice (model 2); 3) whether treatment beliefs, subjective norm, perceived self-efficacy and symptom severity are associated with intended treatment choice (model 3). Path analyses were conducted to examine the hypothesized associations.

Results: 289 patients filled out the booklet. Model 2 had the highest explained variance for each of the treatment modalities (range 32-45\%). Positive treatment beliefs and subjective norm were consistently associated with intended treatment choice across all treatment modalities. Negative treatment beliefs were associated with intended treatment choices for pain medication and arthroplasty. Perceived

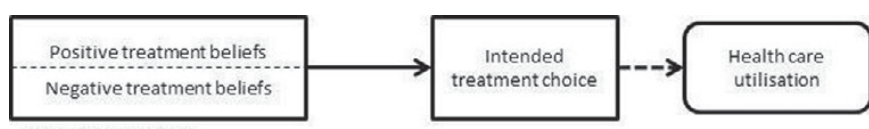

Figure 1a: Model 1

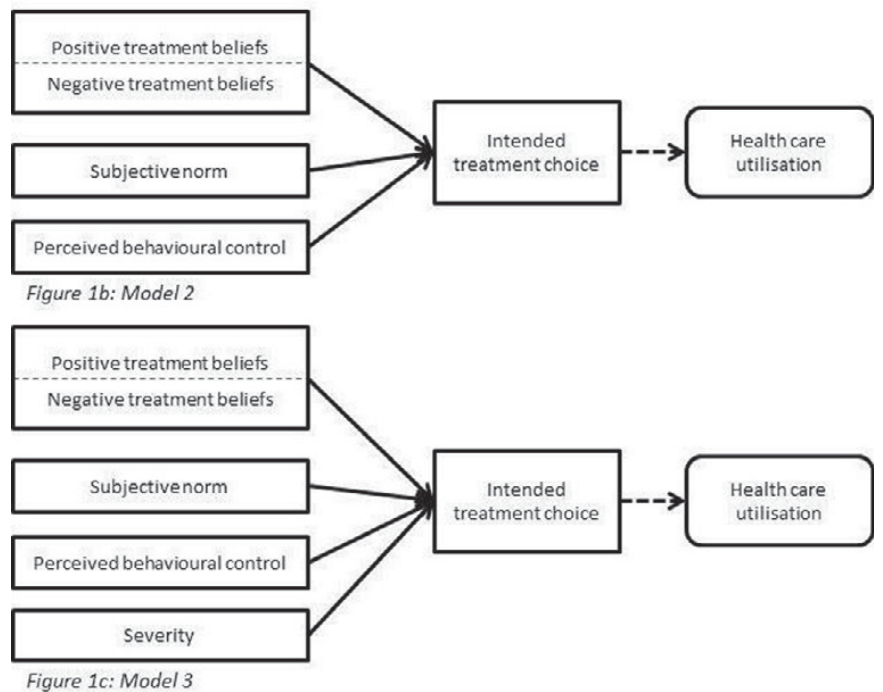

symptom severity was not related to intended treatment choices. No other associations were found.

Conclusions: This is the first study that found empirical support for the relationship between treatment beliefs and treatment choices. The findings suggest that positive beliefs about treatment modalities and the norms and values of one's social environment are related to a specific treatment choice for knee/hip $\mathrm{OA}$ and should be addressed in the clinician's consulting room.

References:

[1] Ajzen, I. (1991). The Theory of Planned Behavior. Organ Behav Hum Dec 50. 179-211.

[2] Rosemann (2007). Health service utilization patterns of primary care patients with osteoarthritis. BMC Health Serv Res 7: 167

Disclosure of Interest: None declared

DOI: 10.1136/annrheumdis-2017-eular.4834

\section{OP0139-HPR REDUCING ARTHRITIS FATIGUE - CLINICAL TEAMS (RAFT) USING COGNITIVE-BEHAVIOURAL APPROACHES: AN RCT}

S. Hewlett ${ }^{1}$, N. Ambler ${ }^{2}$, C. Almeida ${ }^{1}$, P. Blair ${ }^{3}$, E. Choy ${ }^{4}$, E. Dures ${ }^{1}$ W. Hollingworth ${ }^{3}$, B. Kadir ${ }^{3}$, J. Kirwan ${ }^{3}$, Z. Plummer ${ }^{1}$, C. Rooke ${ }^{5}$, J. Thorn ${ }^{3}$, J. Pollock ${ }^{1}$ on behalf of RAFT Study Group. ${ }^{1}$ University of the West of England, Bristol; ${ }^{2}$ Southmead Hospital; ${ }^{3}$ University of Bristol, Bristol; ${ }^{4}$ University of Cardiff, Cardiff; ${ }^{5}$ Bristol Royal Infirmary, Bristol, United Kingdom

Background: RA fatigue is common. Group Cognitive Behavioural Therapy by CBT therapists is effective ${ }^{1}$ but few rheumatology teams have psychologists, thus we trained rheumatology teams to deliver RAFT, a cognitive behavioural approach (CBA).

Objectives: To test if usual care plus a group CBA course for RA fatigue delivered by rheumatology teams reduces fatigue impact more than usual care alone, in a randomised controlled trial.

Methods: A pair of rheumatology nurses/OTs in each of 7 UK hospitals were trained in RAFT. RAFT is 6 , weekly $2 \mathrm{hr}$ group sessions and a consolidation session (wk 14). Links between thoughts, feelings and behaviours (pacing, communication, sleep, stress) are addressed, with daily diaries of energy expenditure and weekly goal-setting. Usual care was a $5 \mathrm{~min}$ discussion of the Arthritis Research UK fatigue booklet. Entry criteria were RA, Bristol RA Fatigue (BRAF-NRS) severity $\geq 6 / 10$ and no recent major medication change. Primary outcome was fatigue impact (BRAF-NRS impact, 0-10) at 26 wks; plus wider aspects of fatigue (BRAF-Multi-Dimensional Questionnaire), pain, disability, sleep, quality of life, mood, self-efficacy, patient global opinion, valued life activities \& disease activity. Intention-to-treat regression analysis involved adjustment for baseline scores and centre.

Results: $308 / 333$ randomized patients completed 26 wks. The 25 who withdrew had similar (10yr) disease duration but were older ( 69 vs $62.4 \mathrm{yrs}$ ). Baseline fatigue impact was similar for RAFT ( $n=156$, BRAF-NRS 7.10, SD 1.7) and controls $(n=152,7.23$, SD 1.6), as were all clinical variables. At 26 wks the RAFT arm had significantly less fatigue impact than controls (BRAF-NRS 5.74 , SD 2.4 vs 6.36 , SD 2.4). Mean BRAF-NRS impact was reduced by $-1.36(p<0.001)$ in RAFT vs -0.88 in controls $(p<0.004)$. Regression analysis showed the difference between changes in fatigue impact NRS was -0.59 in favour of RAFT (Cl -1.11, -0.06).

Regression analysis also showed significant differences in secondary outcomes in favour of RAFT: BRAF-MDQ total fatigue $-3.42(\mathrm{Cl}-6.44,-0.39)$; Living with Fatigue -1.19 (Cl-2.17, -0.21); Emotional Fatique -0.91 (Cl -1.58, -0.23); and RA self-efficacy (RASE, $+3.05, \mathrm{Cl} 0.43,5.66$ ). There were no differences between arms for changes in fatigue severity or other clinical variables.

$99 \%$ of RAFT patients would definitely recommend the course to others compared to $50 \%$ controls $(p<0.001)$. $90 \%$ of RAFT patients rated satisfaction $\geq 8 / 10$ (including $62 \%$ rating $10 / 10$ ); in comparison $50 \%$ controls rated satisfaction $>8 / 10$ (including $26 \%$ rating $10 / 10,(p<0.0001)$. Over 26 weeks 20 control patients sought extra appointments for fatigue help compared to 8 RAFT patients $(14.2 \%$ vs $5.3 \%, p<0.01$.

Conclusions: Rheumatology teams delivering a manualized CBA group intervention addressing fatigue impact, not only improve RA fatigue impact, but also emotional \& overall fatigue, living with fatigue and self-efficacy, with very high patient satisfaction. Providing rheumatology teams with CBA skills is a potential new therapeutic approach to change practice and improve patient outcome. References:

[1] Hewlett et al, ARD 2011;70:1060-7.

Disclosure of Interest: None declared

DOI: 10.1136/annrheumdis-2017-eular.1877

\section{OP0140-HPR ACCEPTANCE AND COMMITMENT THERAPY: A RETROSPECTIVE STUDY OF OUTCOMES FROM A HOSPITAL-BASED, GROUP, PAIN REHABILITATION PROGRAMME IN RHEUMATOLOGY SERVICES IN THE SOUTH EAST OF IRELAND}

N. Nealon Lennox ${ }^{1,2}$, S. O'Neill ${ }^{2}$, A. Hannigan ${ }^{1} .{ }^{1}$ GEMS, University Limerick, Limerick; ${ }^{2}$ Psychology, Ulster University, Derry, Ireland

Background: Acceptance and Commitment Therapy (ACT) is a form of cognitive 